\title{
Severity scoring of lung oedema on the chest radiograph is associated with clinical outcomes in ARDS
}

\author{
Melissa A Warren, ${ }^{1}$ Zhiguou Zhao, ${ }^{2}$ Tatsuki Koyama, ${ }^{2}$ Julie A Bastarache, ${ }^{1}$ \\ Ciara M Shaver, ${ }^{1}$ Matthew W Semler, ${ }_{1}^{1}$ Todd W Rice, ${ }^{1}$ Michael A Matthay, ${ }^{3,4}$ \\ Carolyn S Calfee, ${ }^{3,4}$ Lorraine B Ware ${ }^{1,5}$
}

- Additional material is published online only. To view please visit the journal online (http://dx.doi.org/10.1136/ thoraxjn-2017-211280).

${ }^{1}$ Division of Allergy, Pulmonary and Critical Care Medicine Department of Medicine, Vanderbilt University School of Medicine, Nashville, Tennessee, USA

${ }^{2}$ Department of Boistatistics, Vanderbilt University School of Medicine, Nashville, Tennessee, USA

${ }^{3}$ Department of Medicine,

Cardiovascular Research Institute, University of California, San Francisco, San Francisco, California, USA

${ }^{4}$ Department of Anesthesia, Cardiovascular Research Institute, University of California San Francisco, San Francisco, California, USA

${ }^{5}$ Department of Pathology, Microbiology and Immunology, Vanderbilt University School of Medicine, Nashville, Tennessee, USA

\section{Correspondence to}

Dr Lorraine B Ware, Division of Allergy, Pulmonary and Critical Care Medicine, Department of Medicine, Vanderbilt University School of Medicine, Nashville, TN 37232, USA;

lorraine.ware@vanderbilt.edu

Received 12 November 2017 Revised 8 May 2018 Accepted 14 May 2018 Published Online First 14 June 2018

\section{ABSTRACT}

Background There is no accurate, non-invasive measurement to estimate the degree of pulmonary oedema in acute respiratory distress syndrome (ARDS). We developed the Radiographic Assessment of Lung Oedema (RALE) score to evaluate the extent and density of alveolar opacities on chest radiographs. After first comparing the RALE score to gravimetric assessment of pulmonary oedema in organ donors, we then evaluated the RALE score in patients with ARDS for its relationship to oxygenation and clinical outcomes.

Methods We compared radiographs with excised lung weights from 72 organ donors (derivation cohort) and radiographs with clinical data from 174 patients with ARDS in the ARDSNet Fluid and Catheter Treatment Trial (validation cohort). To calculate RALE, each radiographic quadrant was scored for extent of consolidation (0-4) and density of opacification (1-3). The product of the consolidation and density scores for each of the four quadrants was summed (maximum score $=48$ ).

Results Agreement between two independent reviewers for RALE score was excellent (intraclass correlation coefficient $=0.93,95 \% \mathrm{Cl} 0.91$ to 0.95 ). In donors, pre-procurement RALE score correlated with height-adjusted total lung weight $(\rho=0.59, p<0.001)$. In patients with ARDS, higher RALE scores were independently associated with lower $\mathrm{PaO}_{2}$ /fractional inspired oxygen and worse survival. Conservative fluid management significantly decreased RALE score over 3 days compared with liberal fluid management.

Conclusions The RALE score can be used to assess both the extent of pulmonary oedema and the severity of ARDS, by utilising information that is already obtained routinely, safely and inexpensively in every patient with ARDS. This novel non-invasive measure should be useful for assessing ARDS severity and monitoring response to therapy.

\section{INTRODUCTION}

Pulmonary oedema is a key feature of the pathogenesis and prognosis of acute respiratory distress syndrome (ARDS) ${ }^{1}$ but the severity of pulmonary oedema is only indirectly assessed in current definitions of ARDS by the degree of hypoxaemia. ${ }^{2}$ Current methods to quantify the severity of pulmonary oedema are either invasive (eg, pulse index

\section{Key messages}

What is the key question?

- We asked whether the chest radiograph can be systematically scored to quantify the severity of pulmonary oedema in acute respiratory distress syndrome (ARDS) and whether this radiographic score is associated with the severity of ARDS and clinical outcomes.

What is the bottom line?

- The Radiographic Assessment of Lung Oedema (RALE) score was independently associated with severity of ARDS as assessed by oxygenation and clinical outcomes including mortality.

Why read on?

- The RALE score is a simple non-invasive measure that can be used to assess the severity of ARDS utilising information that is already obtained routinely, safely and inexpensively in every patient with ARDS.

contour continuous cardiac output catheter) or require patient transport out of the intensive care unit (eg, CT imaging), raising safety concerns. We hypothesised that the chest radiograph, already used routinely to detect the presence of ARDS, can be systematically scored to quantify the severity of pulmonary oedema. We further hypothesised that the severity of pulmonary oedema as assessed on the routine chest radiograph is associated with the severity of ARDS and clinical outcomes.

To test these two hypotheses, we developed a novel Radiographic Assessment of Lung Oedema (RALE) score that assesses both the extent and density of alveolar opacities on the chest radiograph. After establishing that the RALE score correlates with the degree of pulmonary oedema by comparing pre-procurement chest radiographs in deceased organ donors to gravimetric measurement of pulmonary oedema in excised lungs, we then applied the RALE score to radiographs from patients with ARDS enrolled in the National Heart, Lung, and Blood Institute (NHLBI) Fluid and Catheter Treatment Trial (FACTT). ${ }^{3}$ 


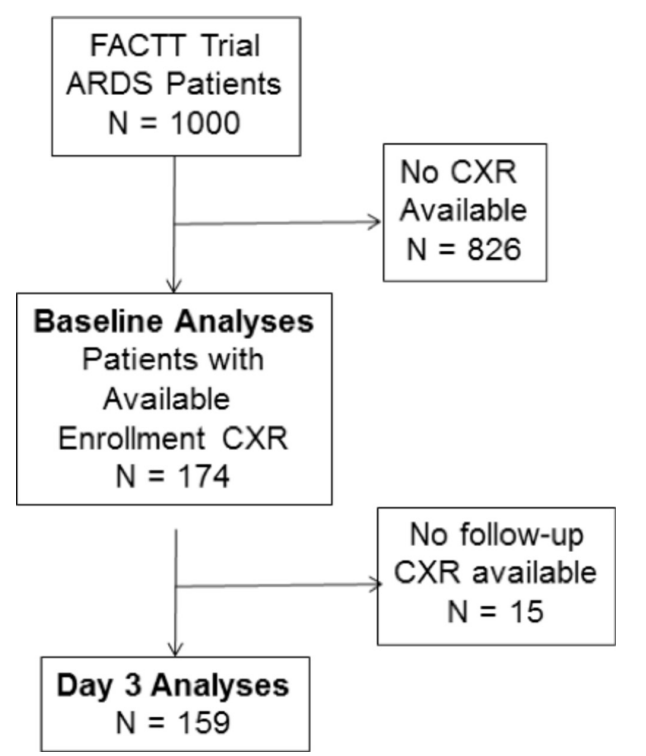

Figure 1 Flow diagram of patients included in the baseline (time of enrolment) analyses and the day 3 analyses of the RALE score. Day 3 analyses were done using chest radiographs from day 3 after enrolment when available $(n=140)$. If a chest radiograph was not available from day 3 , a chest radiograph from the next closest day was used with a preference for day 4 if available (day $1, n=1$; day $2, n=3$; day $4, n=14$; day $6, n=1$ ). ARDS, acute respiratory distress syndrome; CXR, chest radiograph; FACTT, Fluid and Catheter Treatment Trial; RALE, Radiographic Assessment of Lung Oedema.

\section{MATERIALS AND METHODS \\ Patient populations}

The initial evaluation of the RALE score was done using chest radiographs and gravimetric measurements of lung oedema from a derivation cohort of donors enrolled in the Beta Agonist for Oxygenation in Lung Donors (BOLD) study, a randomised clinical trial of nebulised albuterol in deceased organ donors. ${ }^{4}$ Following organ resection without perfusion, lungs from enrolled donors declined for transplantation were weighed. Total lung weights were adjusted for patient height and used as a quantitative index of pulmonary oedema. ${ }^{5}$ Included in the study were 72 of 506 donors in BOLD who had matched preprocurement radiographs and lung weights.

To assess clinical utility of RALE, a validation cohort of patients from the NHLBI ARDS Network FACTT were studied. ${ }^{3}$ FACTT was a multicenter, randomised trial of conservative versus liberal fluid management in 1000 patients with ARDS. Patients $(n=174)$ were included from five FACTT sites if enrolment chest radiographs were available for scoring; these patients were previously included in a study of vascular pedicle width. ${ }^{6}$ Patient selection is summarised in figure 1. Treatment arm, baseline $\mathrm{PaO}_{2} /$ fractional inspired oxygen $\left(\mathrm{FiO}_{2}\right)$, fluid balance and clinical outcomes including ventilator-free days (VFDs) and survival were obtained from the trial database.

\section{Radiograph scoring}

Details of the RALE score are provided in figure 2. To determine the RALE score, each radiograph was divided into quadrants, defined vertically by the vertebral column and horizontally by the first branch of the left main bronchus. Each quadrant was assigned a consolidation score from 0 to 4 to quantify the extent of alveolar opacities, based on the percentage of the quadrant with opacification and a density score from 1 to 3 to quantify the overall density of alveolar opacities, unless the consolidation score for that quadrant was 0 . The density score $(1=$ hazy, $2=$ moderate, $3=$ dense) allows for more quantitative assessment of the density of opacification by quadrant. To calculate the final RALE score, the product of the consolidation and density score for each quadrant were summed for a final RALE score ranging from 0 (no infiltrates) to 48 (dense consolidation in $>75 \%$ of each quadrant).
A

\begin{tabular}{|c|c|}
\hline \multicolumn{2}{|c|}{ Consolidation $^{2}$} \\
\hline Consolidation Score & Extent of alveolar opacities \\
\hline $\mathbf{0}$ & None \\
\hline 1 & $<25 \%$ \\
\hline 2 & $25-50 \%$ \\
\hline 3 & $50-75 \%$ \\
\hline 4 & $>75 \%$ \\
\hline \multicolumn{2}{|c|}{ Density ${ }^{b}$} \\
\hline $\begin{array}{c}\text { Density } \\
\text { Score }\end{array}$ & Density of alveolar opacities \\
\hline 1 & Hazy \\
\hline 2 & Moderate \\
\hline 3 & Dense \\
\hline \multicolumn{2}{|c|}{ Final RALE Score ${ }^{\mathrm{c}}$} \\
\hline Right Lung & Left Lung \\
\hline Upper Quadrant & Upper Quadrant \\
\hline Cons $x$ Den $=\mathrm{Q} 1$ score & Cons $x$ Den $=Q 3$ score \\
\hline Lower Quadrant & Lower Quadrant \\
\hline Cons $x$ Den $=\mathrm{Q} 2$ score & Cons $\times$ Den $=Q 4$ score \\
\hline \multicolumn{2}{|c|}{ Total RALE $=\mathrm{Q} 1+\mathrm{Q} 2+\mathrm{Q3}+\mathrm{Q4}$} \\
\hline
\end{tabular}

${ }^{3}$ Consolidation is scored for each quadrant

"Consolidation is scored for each quadrant
bensity is scored for each quadrant that has a consolidation score $\geq 1$

If Quadrant consolidation score is - then Quadrant score is 0
B

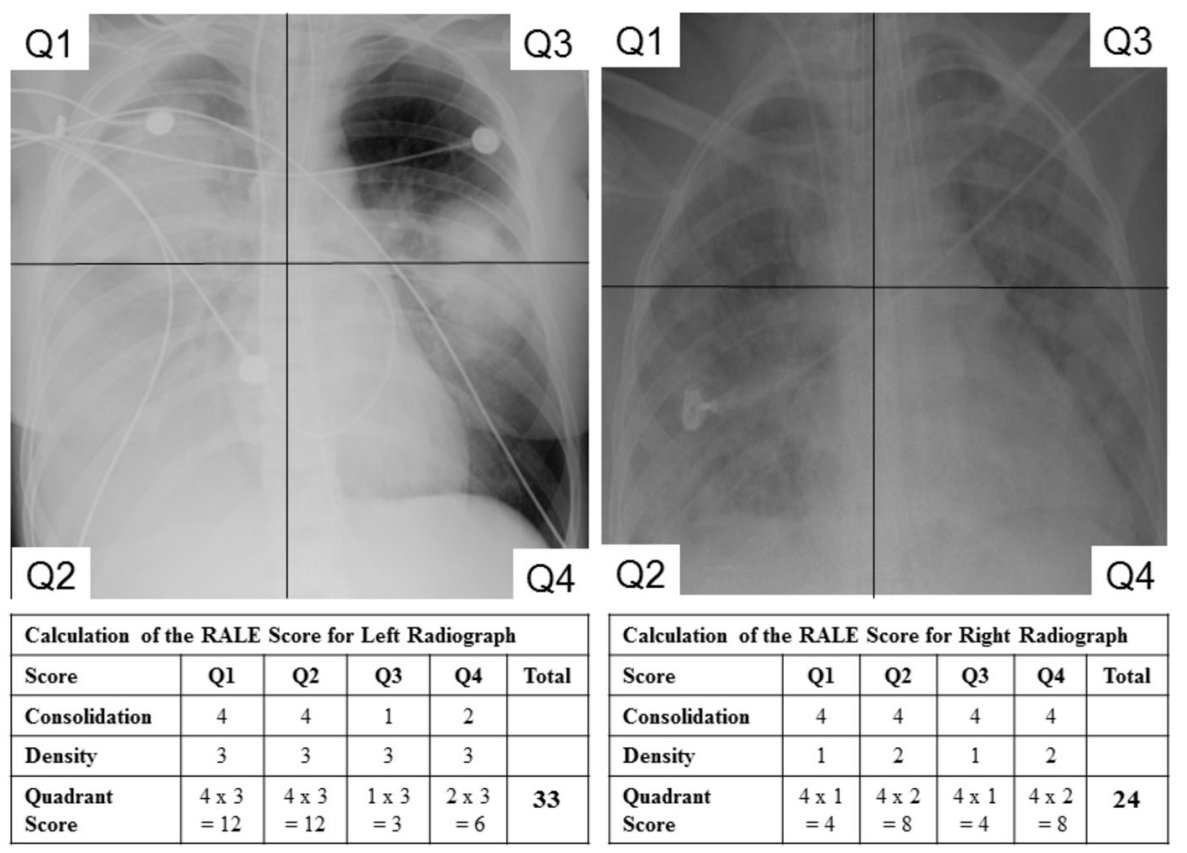

Figure 2 Consolidation and density scoring in the Radiographic Assessment of Lung Oedema (RALE) score (panel A). Calculation of the RALE score (panel B). 
In the organ donors, chest radiographs obtained within 24 hours of lung procurement were scored for each donor, using the radiograph obtained closest to the time of procurement. Radiographs were scored by consensus of two physician reviewers without knowledge of clinical data or lung weight. In FACTT, each radiograph was scored independently by two physician reviewers blinded to treatment arm, in order to examine interobserver reliability.

\section{Statistical analysis}

To determine how well the RALE score quantifies pulmonary oedema, we evaluated the association between preprocurement RALE score and total excised lung weight in deceased organ donors using Spearman's correlations. A subgroup of 36 donors with total lung weight above the median $(797 \mathrm{~g})$ was used to assess the utility of the RALE score in donors with more pulmonary oedema.

To assess the reliability of RALE score across independent reviewers, a two-way mixed consistency, average-measures intraclass correlation coefficient (ICC) ${ }^{7}$ was calculated for baseline and day 3 RALE scores in the FACTT trial. Bland-Altman plots were used to visualise agreement between independent reviewers.

The clinical utility of the RALE score was assessed using chest radiographs and clinical data from the FACTT trial. To assess the association with ARDS severity, RALE scores were compared with baseline $\mathrm{PaO}_{2} / \mathrm{FiO}_{2}$ using multivariable linear regression. Associations between RALE and other clinical outcomes (overall survival, 28-day mortality, 60-day mortality and VFDs) were also evaluated. For survival, a Cox Proportional Hazard model was fit. Kaplan-Meier estimates were computed for groups defined by baseline RALE score quartiles. For 28-day mortality and 60-day mortality, logistic regression models were used. For VFDs, zero-inflated negative binomial models were used to account for the high frequency of patients with zero VFDs.

To assess the potential value of change in RALE as a study endpoint, we evaluated the association between change in RALE from baseline to day 3 and FACTT treatment arm (conservative or liberal fluid management) in regression models adjusted for age, gender, body mass index (BMI) and acute physiology and chronic health evaluation III (APACHE III). We also used linear regression with fluid balance as the dependent variable and RALE, age, gender, BMI and APACHE III as covariates to assess the relationship between RALE and fluid balance.

Continuous variables are summarised with median and IQR. Categorical variables are summarised as frequencies with percentages. Differences between groups were assessed using Wilcoxon rank sum (continuous) and Fisher's exact test (categorical). For linear regression and Cox proportional hazard models, we report the estimated effect associated with a fiveunit change in RALE score. Statistical significance was considered when $\mathrm{p}<0.05$. Analyses were performed using R V.3.3.1. ${ }^{8}$

\section{RESULTS \\ Validation of the RALE score against gravimetric measurement of lung oedema}

Characteristics of deceased organ donors are given in the online supplementary table 1 . The preprocurement RALE score was significantly correlated with total lung weight $(\rho=0.59$, $\mathrm{p}<0.001$ ) (online supplementary figure 1 ). Because the extent of pulmonary oedema in organ donors is typically lower than in ARDS, we also evaluated the subgroup with more pulmonary oedema, as evidenced by lung weight above the median $(n=36)$;
Table 1 Clinical characteristics of patients from Fluid and Catheter Treatment Trial $(n=174)$ who were included in the current study by treatment arm, compared with those who were not included in the current study

\begin{tabular}{llllc}
\hline $\begin{array}{l}\text { Clinical } \\
\text { characteristics }\end{array}$ & $\begin{array}{l}\text { Included } \\
\text { conservative fluid } \\
\text { arm }(\mathbf{n}=82)\end{array}$ & $\begin{array}{l}\text { Included } \\
\text { liberal fluid } \\
\text { arm }(\mathbf{n}=92)\end{array}$ & P values* & $\begin{array}{l}\text { Not included } \\
(\mathbf{n}=826)\end{array}$ \\
\hline Age & $48(39,59)$ & $44(35,60)$ & 0.70 & $49(39,61)$ \\
\hline Male & $44(54 \%)$ & $42(46 \%)$ & 0.29 & $448(54 \%)$ \\
\hline Caucasian & $64(79 \%)$ & $62(68 \%)$ & 0.05 & $513(62 \%)$ \\
\hline BMI $\left(\mathrm{kg} / \mathrm{m}^{2}\right)$ & $28(25,34)$ & $28(23,34)$ & 0.59 & $27(23,32)$ \\
\hline APACHE III & $89(74,105)$ & $95(81,121)$ & 0.07 & $91(69,117)$ \\
\hline $60-$ day mortality & $18(22 \%)$ & $26(28 \%)$ & 0.34 & $225(27 \%)$ \\
\hline RALE baseline & $28(17,35)$ & $26(20,34)$ & 0.64 & NA \\
\hline RALE day 3 & $16(10,23)$ & $25(16,35)$ & $<0.001$ & NA \\
\hline Change in RALE† & $-8(-1,-16)$ & $-1(-8,5)$ & $<0.001$ & NA \\
\hline
\end{tabular}

Data as median and IQR.

${ }^{*} \mathrm{P}$ value is for comparison between patients in the conservative and liberal fluid management arms who were included in the current study.

tChange in RALE from baseline radiograph to day 3 radiograph. Negative number indicates that the score decreased from baseline to day 3 .

APACHE III, acute physiology and chronic health evaluation III; BMI, body mass index; NA, not available; RALE, radiographic assessment of lung oedema.

the correlation was higher in this subgroup (online supplementary figure 1$)(\rho=0.73, p<0.001)$.

\section{Association of RALE scoring with clinical outcomes in ARDS}

Characteristics of 174 patients from FACTT are given in table 1. Baseline RALE score was substantially higher than in BOLD (median 27, IQR 18-35 vs 7, IQR 5-15), reflecting the presence of ARDS in FACTT patients. The scores of the two reviewers are compared in figure 3. ICCs were excellent both at baseline (ICC $=0.93$, 95\% CI 0.91 to 0.95 ; figure $3 \mathrm{~A}$ ) and at day 3 (ICC $=0.96,95 \% \mathrm{CI} 0.94$ to 0.97 ; figure $3 \mathrm{~B})$, indicating a high degree of agreement (14). Bland-Altman plots (figure 3C, D) also showed strong agreement across the range of RALE scores.

Lower baseline RALE score (reflecting less pulmonary oedema) was independently associated with better oxygenation as reflected by higher $\mathrm{PaO}_{2} / \mathrm{FiO}_{2}$ - for every five-point decrease in RALE, the baseline $\mathrm{PaO}_{2} / \mathrm{FiO}_{2}$ increased by $8.4 \mathrm{~mm} \mathrm{Hg}$ (95\% CI 3.2 to 13.5 , $\mathrm{p}=0.002)$. Adjusting for age, gender and BMI did not attenuate the association $(\beta=8.4,95 \%$ CI 3.0 to $13.7, p=0.002$, online supplementary table 2). Lower baseline RALE score was also independently associated with better survival, in both unadjusted analysis $(p<0.001)$ and analysis controlling for age, gender, BMI and APACHE III-with every five-point decrease in RALE score, the adjusted hazard of death decreased by $16 \%$ (HR 0.84 ; 95\% CI 0.72 to $0.99, \mathrm{p}=0.032$, online supplementary table 3). Survival curves (figure 4) show that compared with patients with a high baseline RALE score (in third or fourth quartiles), those with a lower baseline RALE score had better survival $(\mathrm{p}<0.001)$. Lower baseline RALE score was also associated with better 28-day mortality (OR 0.76 ; 95\% CI 0.62 to $0.92, \mathrm{p}=0.006)$ and 60-day mortality (OR $0.74 ; 95 \%$ CI 0.61 to $0.89, \mathrm{p}=0.002$ ), in models controlling for age, gender and BMI. These associations were no longer statistically significant after adjusting for APACHE III (OR 0.86; 95\% CI 0.69 to 1.06 , $\mathrm{p}=0.16$ for 28 -day mortality and OR $0.84 ; 95 \%$ CI 0.69 to 1.03 , $\mathrm{p}=0.097$ for 60 -day mortality). For 28 -day mortality, the area under the curve for mortality association with baseline RALE 

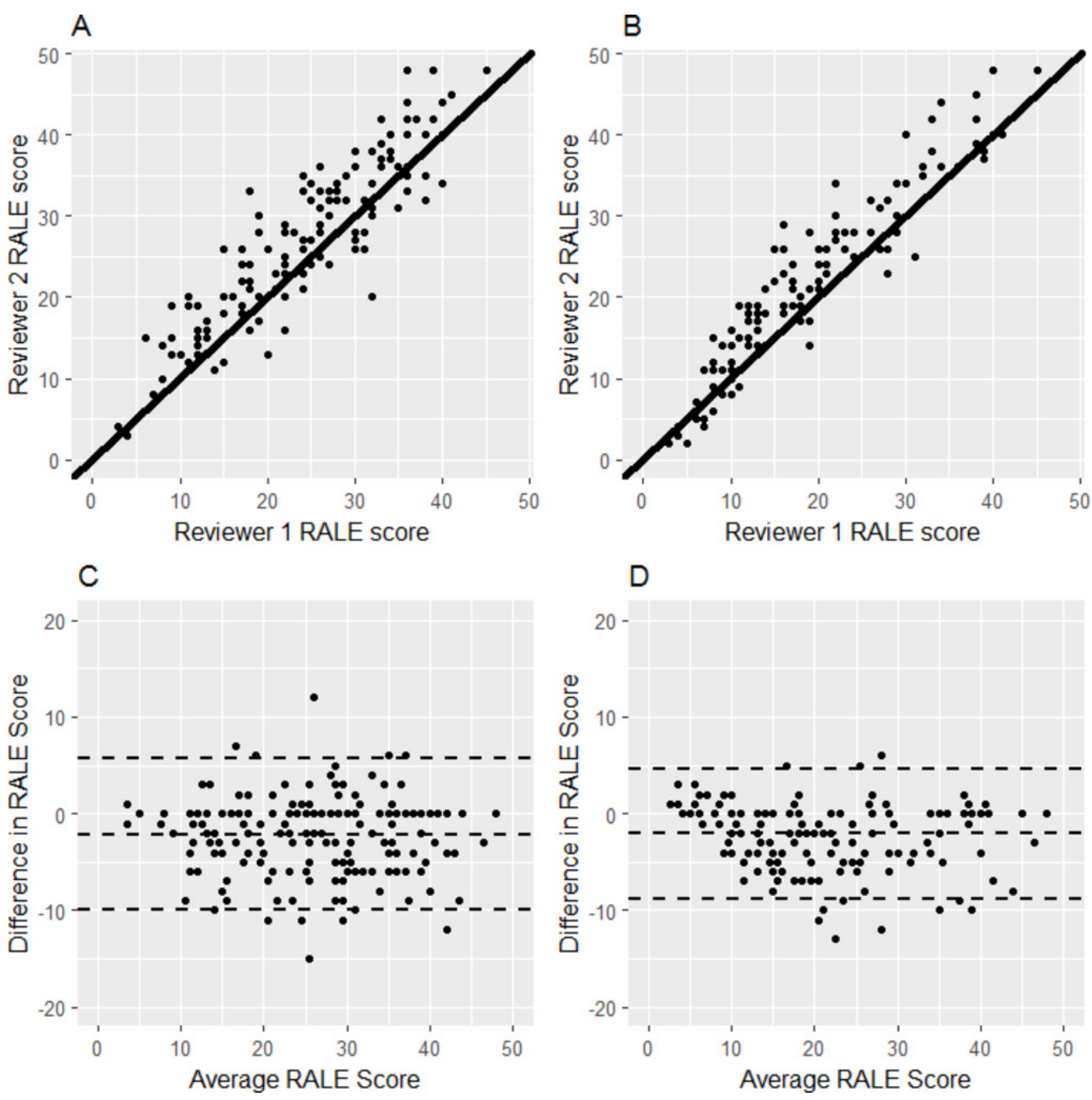

Figure 3 Scatter plots (panels A and B) and Bland-Altmann plots (panels C and D) showing agreement between two independent reviewers for Radiographic Assessment of Lung Oedema (RALE) scores at baseline ( $n=174$, panels A and C) and at day 3 after enrolment ( $n=159$, panels B and D) for patients in the Fluid and Catheter Treatment Trial. To assess agreement between the two reviewers, intraclass correlation coefficients (ICCs) were calculated. ICCs were excellent both at baseline (ICC $=0.93,95 \% \mathrm{Cl} 0.91$ to 0.95$)$ and at day $3(\mathrm{ICC}=0.96,95 \% \mathrm{Cl} 0.94$ to 0.97$)$.

score was $0.82(95 \%$ CI 0.74 to $0.90, \mathrm{p}<0.001)$. Baseline RALE score was not associated with VFDs $(\mathrm{p}=0.59)$.

RALE scores at baseline were not significantly different between liberal and conservative fluid arms (table 1). However, day 3 RALE score was significantly lower in the conservative arm compared with the liberal arm (table $1 ; \mathrm{p}<0.001$ ). From baseline to day 3, the RALE score declined significantly more in the conservative fluid arm than the liberal arm (table 1 and figure 5A). This treatment effect remained significant in multivariable analysis $(\beta=6.98,95 \% \mathrm{CI} 3.73$ to $10.22, \mathrm{p}<0.001)$. Furthermore, a decrease in RALE score from baseline to day 3 was associated with lower cumulative fluid balance. Specifically, for every five-point decrease in RALE score, cumulative fluid balance decreased by $0.85 \mathrm{~L}(95 \% \mathrm{CI} 0.18$ to $1.53, \mathrm{p}=0.01)$ (figure 5B).

\section{DISCUSSION}

The primary objectives of this study were to determine whether the chest radiograph can be systematically scored to quantify the severity of pulmonary oedema and to determine whether this radiographic scoring is associated with the severity of ARDS and clinical outcomes. To this end, we developed the RALE score and tested it in deceased organ donors and in patients with ARDS.
In deceased organ donors, preprocurement RALE scores correlated with total excised lung weights, evidence that the RALE score provides a quantitative index of lung oedema. In patients with ARDS, there was a significant, independent correlation between higher baseline RALE score and lower $\mathrm{PaO}_{2} / \mathrm{FiO}_{2}$. The RALE score was also strongly and independently associated with ARDS outcomes including overall survival and 28-day mortality and 90-day mortality. Calculation of the score is simple and was robustly reproducible when independent readings were compared. Taken together, these findings indicate that the RALE score provides a clinically significant assessment of the extent of pulmonary oedema in patients with ARDS that is reflected both by the severity of hypoxaemia and by adverse clinical outcomes. Furthermore, the RALE score declined in response to treatment targeted at reducing pulmonary oedema (the conservative fluid arm of FACTT), suggesting potential value both for assessment of therapeutic response and as a radiographic clinical outcome.

There are multiple potential applications for the RALE score in patients with ARDS both for patient care and for clinical research. RALE scores could be used for clinical identification of patients at highest risk of mortality, leading to earlier detection, intervention and treatment of high-risk patients with ARDS. A similar approach could be used for 


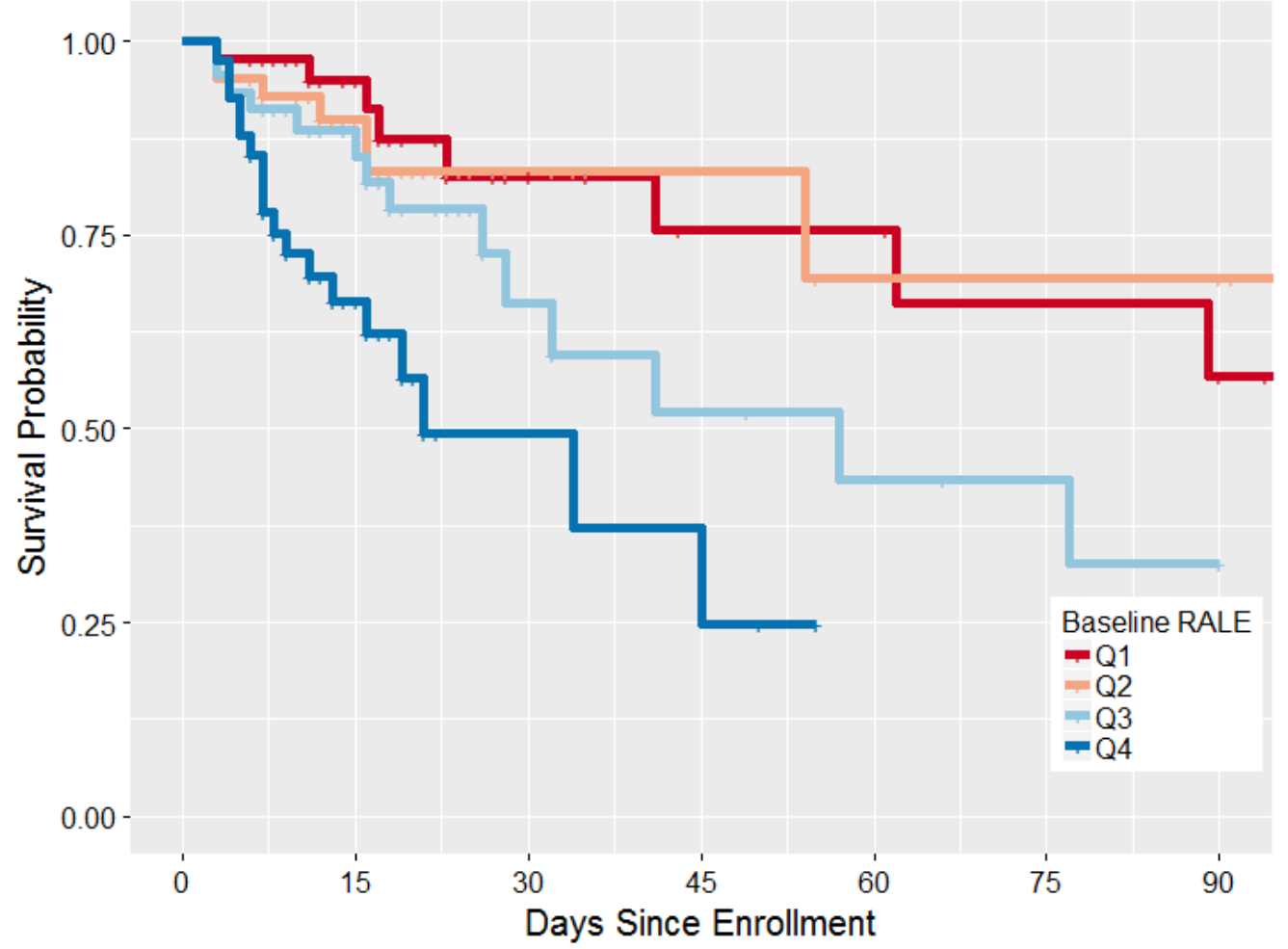

Number at risk

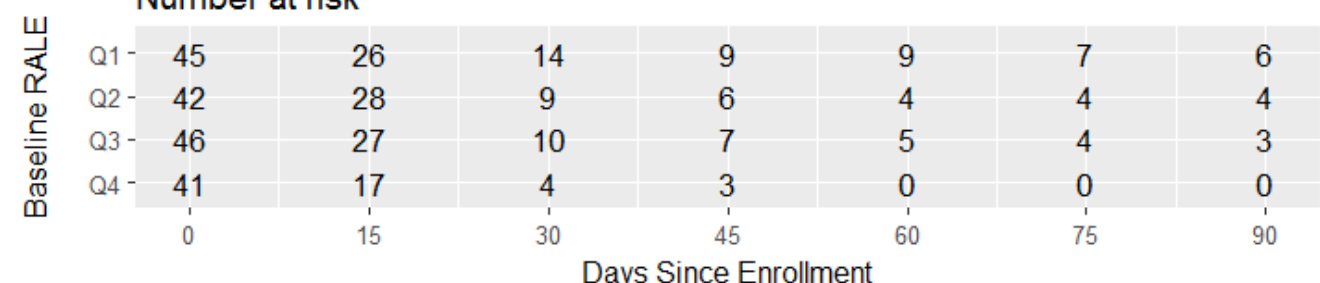

Figure 4 Kaplan-Meier estimates of acute respiratory distress syndrome (ARDS) survival stratified by the baseline Radiographic Assessment of Lung Oedema (RALE) score for patients in Fluid and Catheter Treatment Trial. ARDS survival was significantly lower in patients with baseline RALE score above the median (third and fourth quartiles, $\mathrm{p}<0.001$ ). Quartile 1, Q1; Quartile 2, Q2; Quartile 3, Q3; Quartile 4, Q4.

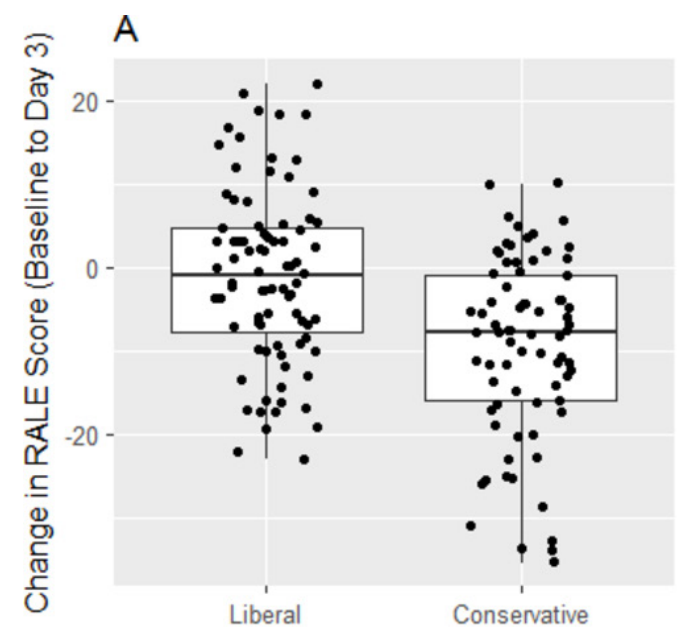

Fluid Management

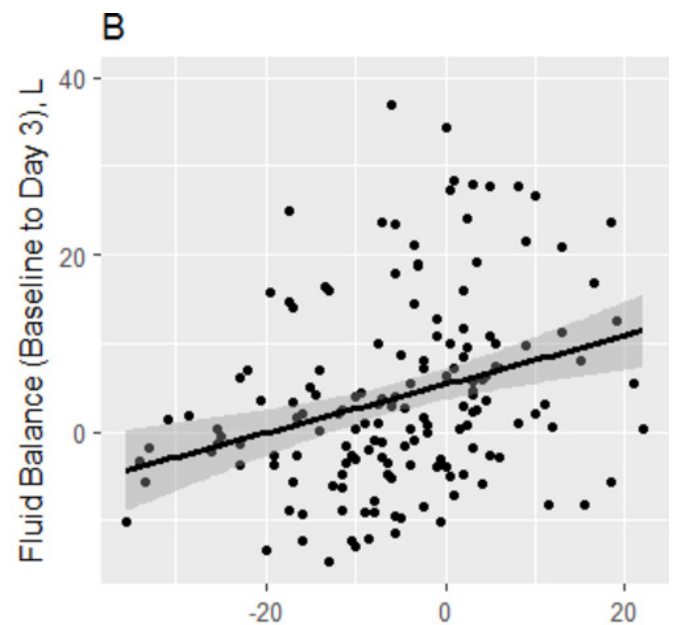

Change in RALE Score (Baseline to Day 3)

Figure 5 The change in Radiographic Assessment of Lung Oedema (RALE) score differed by treatment arm in Fluid and Catheter Treatment Trial, with a significant drop in score from enrolment to day 3 in the conservative fluid management arm compared with the liberal fluid management arm (panel A). This treatment effect remained significant in multivariable analysis ( $\beta=6.98,95 \% \mathrm{Cl} 3.73$ to $10.22, p<0.001)$. Independent of the treatment arm, the change in RALE score from enrolment to day 3 was significantly associated with fluid balance (panel B). Specifically, for every five-point decrease in RALE score, cumulative fluid balance decreased by $0.85 \mathrm{~L}(95 \% \mathrm{Cl} 0.18$ to $1.53, \mathrm{p}=0.01)$. 
risk stratification for enrolment of patients with ARDS into therapeutic clinical trials. Assessment of ARDS severity has been recently used with some success to enrich clinical trials for the more severely ill, ${ }^{9}{ }^{10}$ but the only severity assessment used to date has been the $\mathrm{PaO}_{2} / \mathrm{FiO}_{2}$ ratio. Since chest radiographs are routinely obtained in patients with ARDS, use of RALE for risk stratification would not require additional testing or cost and could be done without delay in all patients, providing additional information about ARDS severity beyond that provided by the $\mathrm{PaO}_{2} / \mathrm{FiO}_{2}$ ratio. Serial assessment of RALE scores could be used to assess response to treatment clinically, or as an endpoint in clinical trials.

To our knowledge, apart from a prior study of a radiographic consolidation score that was used as a clinical outcome in the BOLD clinical trial in deceased organ donors, ${ }^{11}$ the current study is the only human study that compares a chest radiographic score to the quantitative assessment of pulmonary oedema by direct measurement of lung weight. Several other studies comparing a simpler radiographic score to extravascular lung water (EVLW) measured by single-indicator or double-indicator methods were limited in the ability to show reliable correlation, perhaps due to limitations of prior radiographic scoring methods. ${ }^{12-14}$ Both computerised axial tomography and ultrasound have been used to assess the presence and distribution of lung oedema, ${ }^{15}$ although neither would be as practical as RALE scoring for daily assessment. However, it would be potentially valuable in the future to compare ultrasound assessments with RALE score since like chest radiography, lung ultrasound can be done non-invasively at the bedside. The only other semiquantitative score that has been used to assess the severity of pulmonary oedema on the chest radiograph in patients with ARDS is the radiographic component of the Murray Lung Injury Score (LIS) ${ }^{16}$ However, the radiographic component of the LIS provides very little discrimination, only scoring each quadrant for the presence or absence of opacities. In a study of the prognostic value of the LIS in ARDS, the radiographic component of the LIS provided virtually no discrimination of outcomes compared with the other components of the LIS. ${ }^{17}$

This study has several strengths. First, in contrast to prior studies that estimated the extent of pulmonary oedema by EVLW, we directly quantified the degree of pulmonary oedema using total lung weight in donor lungs. Second, in FACTT, chest radiographs were independently scored by two clinician investigators to assess interobserver reliability. The high level of agreement supports the potential applicability of the RALE score for clinicians at the bedside. Third, patients from FACTT originated from multiple different study sites and had a variety of underlying causes of ARDS, supporting broad applicability of the findings.

There are also some limitations. We were only able to study a subset of patients from the FACTT trial due to limited availability of chest radiographs. This limited our power to explore some aspects of the association between RALE score and ARDS severity and outcomes such as non-linear associations. Despite this, we found clinically meaningful associations between RALE score and oxygenation, clinical outcomes and fluid management. Because the FACTT patients were derived from a clinical trial with highly selective inclusion and exclusion criteria, future studies of the RALE score in ARDS should include a broader population of patients to enhance generalisability. Patients with trauma were under-represented in the FACTT cohort, so the applicability of the current findings to trauma-associated
ARDS is uncertain. Also, application of the RALE score may be challenging in patients who have extensive atelectasis, pleural effusions or morbid obesity. Furthermore, the level of positive end-expiratory pressure (PEEP) could affect the RALE score. ${ }^{18}$ Although PEEP levels were protocolised in FACTT, this is an important area for further study. Finally, it will be important to validate the RALE score in other clinical setting and data sets and to further investigate the utility of the scoring system in comparison to lung ultrasonography and CT imaging in the assessment of the extent of pulmonary oedema.

\section{CONCLUSIONS}

Radiographic assessment of the extent of pulmonary oedema using the RALE score correlates well with direct gravimetric assessment of pulmonary oedema. In patients with ARDS, the RALE score was independently association with both ARDS severity, response to conservative fluid management and clinical outcomes. Taken together, these findings suggest that the RALE score provides an innovative new method to leverage information that is already collected routinely in patients with ARDS to non-invasively assess both the extent of pulmonary oedema and the severity of ARDS.

Acknowledgements We would like to acknowledge the BOLD study investigators and the NHLBI ARDSNet FACTT trial investigators for collection of the primary clinical data that was analysed in this study.

Contributors MAW and LBW contributed to data collection. MAW, ZZ, TK and LBW contributed to figures. MAW, JAB, CMS, MWS, TWR, MAM, CSC and LBW contributed to the study design. All authors contributed to data analysis, data interpretation and writing of the manuscript.

Funding Funding for this study was provided by NIH HL103836, R37 HL51856, HL131621, HL126671, Courtney's Race for the ARDS Cure and the Courtney Charneco Family.

Competing interests $J A B$ has received research funding from the National Institutes of Health and Global Blood Therapeutics. TWR has received consulting fees from Avisa Pharma and Cumberland Pharmaceuticals. CSC has received research funding from the National Institutes of Health, the Food and Drug Administration (FDA), the Department of Defense, Bayer and GlaxoSmithKline. She has received consulting fees from GlaxoSmithKline, Boehringer Ingelheim, Bayer, Prometic, CSL Behring and Roche/Genentech. MAM has received research grants from Amgen and GlaxoSmithKline and has a current research grant from Bayer Pharmaceuticals for ARDS studies. He has received consultation fees from CSL Behring, Boehinger Ingelheim, Cerus Therapeutics, Quark Pharmaceuticals, Thesan Pharmaceuticals and Bayer Pharmaceuticals. He was Chair of a DSMB for Asthma trials for RocheGenentech (2013-2017). He also receives grant support for research and clinical trials from the NHLBI, FDA and the Department of Defense. LBW has received research funding from the National Institutes of Health, Boehringer Ingelheim and Global Blood Therapeutics and consultant fees from CSL Behring.

Patient consent Not required.

Ethics approval Institutional IRBs at all FACTT study sites.

Provenance and peer review Not commissioned; externally peer reviewed.

(c) Article author(s) (or their employer(s) unless otherwise stated in the text of the article) 2018. All rights reserved. No commercial use is permitted unless otherwise expressly granted.

\section{REFERENCES}

1 Matthay MA, Ware LB, Zimmerman GA. The acute respiratory distress syndrome. J Clin Invest 2012;122:2731-40.

2 Ranieri VM, Rubenfeld GD, Thompson BT, et al. Acute respiratory distress syndrome: the Berlin Definition. JAMA 2012;307:2526-33.

3 Wiedemann HP, Wheeler AP, Bernard GR, et al. Comparison of two fluid-management strategies in acute lung injury. N Engl J Med 2006;354:909-75.

4 Ware $L B$, Landeck $M$, Koyama $T$, et al. A randomized trial of the effects of nebulized albuterol on pulmonary edema in brain-dead organ donors. Am J Transplant 2014; 14:621-8.

5 Staub NC. Pulmonary edema. Physiol Rev 1974;54:678-811.

6 Rice TW, Ware LB, Haponik EF, et al. Vascular pedicle width in acute lung injury: correlation with intravascular pressures and ability to discriminate fluid status. Crit Care 2011;15:R86 
7 McGraw KO, Wong SP. Forming inferences about some intraclass correlation coefficients. Psychol Methods 1996;1:30-46.

8 R: a language and environment for statistical computing. Vienna, Austria: R Foundation for Statistical Computing, 2016.

9 Guérin C, Reignier J, Richard JC, et al. Prone positioning in severe acute respiratory distress syndrome. N Engl J Med 2013;368:2159-68.

10 Papazian L, Forel JM, Gacouin A, et al. Neuromuscular blockers in early acute respiratory distress syndrome. N Engl J Med 2010;363:1107-16.

11 Ware LB, Neyrinck A, O'Neal HR, et al. Comparison of chest radiograph scoring to lung weight as a quantitative index of pulmonary edema in organ donors. Clin Transplant 2012;26:665-71.

12 Sivak ED, Richmond BJ, O'Donavan PB, et al. Value of extravascular lung water measurement vs portable chest $\mathrm{x}$-ray in the management of pulmonary edema. Crit Care Med 1983;11:498-501.
13 Halperin BD, Feeley TW, Mihm FG, et al. Evaluation of the portable chest roentgenogram for quantitating extravascular lung water in critically ill adults. Chest 1985;88:649-52.

14 Brown LM, Calfee CS, Howard JP, et al. Comparison of thermodilution measured extravascular lung water with chest radiographic assessment of pulmonary oedema in patients with acute lung injury. Ann Intensive Care 2013;3:25.

15 Bellani G, Rouby JJ, Constantin JM, et al. Looking closer at acute respiratory distress syndrome: the role of advanced imaging techniques. Curr Opin Crit Care 2017;23:30-7.

16 Murray JF, Matthay MA, Luce JM, et al. An expanded definition of the adult respiratory distress syndrome. Am Rev Respir Dis 1988;138:720-3.

17 Kangelaris KN, Calfee CS, May AK, et al. Is there still a role for the lung injury score in the era of the Berlin definition ARDS? Ann Intensive Care 2014;4:4.

18 Wallet $F$, Delannoy B, Haquin A, et al. Evaluation of recruited lung volume at inspiratory plateau pressure with PEEP using bedside digital chest $\mathrm{X}$-ray in patients with acute lung injury/ARDS. Respir Care 2013;58:416-23. 